\title{
Mean Tube Current
}

National Cancer Institute

\section{Source}

National Cancer Institute. Mean Tube Current. NCI Thesaurus. Code C69233.

The mean value of the electrical current applied to an X-ray tube. 